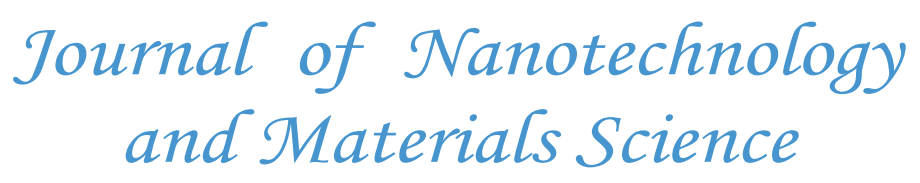

\title{
Designing Nanomaterials for Multifunctional Applications
}

\author{
Jai Prakash* \\ Department of Chemical Engineering, Indian Institute of Technology (IIT) Kanpur, Kanpur-208016, UP, India
}

*Corresponding author: Jai Prakash, INSPIRE Faculty, Department of Chemical Engineering, Indian Institute of Technology, Kanpur, Kanpur-208016, India (At present) and Visiting Research Fellow, Department of Physics, University of the Free State (UFS), Bloemfontein, 9300, South Africa, E-mail: jaip@iitk.ac.inb, jai.gupta1983@gmail.com, prakashJ@ufs.ac.za

Citation: Prakash, J. Designing Nanomaterials for Multifunctional Applications. (2016) J Nanotech Mater Sci 3(2): 41- 42.

It is an honor to write on editorial text for Journal of Nanotechnology and Material Science which covers all aspect of materials science and nanotechnology with open access facility with an attempt to reach each and every reader. In this editorial, I would focus on role of functional nanomaterials and their utilization in broad range of applications. As the name of journal itself indicates, using nanotechnology, how the materials can be synthesized and used involving all branches of the science. It is possible by designing multifunctional nanomaterials which involve interdisciplinary research including all field of scientific research.

Significant research has been carried out in materials science during last decades to design variety of nanomaterials and tailoring/engineering their properties for advanced functional applications. Nanomaterials have demonstrated their applications in a variety of aspects ranging from basic science to medicine, electronics, environment, energy and biomedical applications. Nanotechnology has provided several innovative methods, developed various techniques and shown future prospects for synthesizing functional nanomaterials and their effective applications in almost every step of life. Moreover, it has ability to control the material's structures, their properties and provides fundamental understanding of occurrence taking place at nanoscale level.

Recent developments in the field of nanotechnology studying nanostructures and their interfacial interaction with materials of different functionalities have encouraged the scientific community to develop nanomaterials with multifunctional application. Nanocomposites are such a class of multifunctional nanomaterials which are generally produced by coupling dissim-

\author{
Received date: November 15, 2016 \\ Accepted date: November 18, 2016 \\ Published date: November 28, 2016
}

ilar nanostructured materials providing new class of materials with modified structures and enhanced properties than that of the parent materials ${ }^{[1]}$. The study of these nanocomposite materials not only provides a specific nanomaterial with multifunctional behaviors but also gives fundamental understanding of interdisciplinary research amongst the various fields of sciences such as physics, chemistry and biology of nanomaterials. These nanocomposite multifunctional materials can be composed of any two or more functional nanomaterials with improved properties in form of a compound for an application or can be of multifunctional applications to be used in variety of fields due to their synergistic contributions. For example: Noble Metal Nanoparticles (NMNPs) when form composite with other nanomaterials such as magnetic, carbon based nanomaterials (graphene, carbon nanotubes, fullerenes), polymers, metal oxides etc., new nanocomposite materials thus formed exhibit novel magnetic, electrical and semiconducting properties along with their unique optical properties based on surface plasmon resonance characteristics $^{[1-3]}$. These nanocomposite materials with multifunctional elements are useful in various fields such as sensors, electricals/ electronics, energy, environments and biomedical engineering etc. NMNPs based nanocomposite nanomaterials have been extensively studied in the past decades because of their unique optical properties. Nanocomposites of NMNPs with metal oxides have been shown to have promising multifunctional characteristics and multifunctional applications in the field of antibacterial, photocatalyst, sensors and detection of traces using surface enhanced Raman scattering nanotechnology for environmental as well as biomedical applications ${ }^{[1]}$. On the other hand, nanocomposites of NMNPs with carbon nanomaterials have been 
synthesized for multifunctional application in various fields such as electronics, biosensing as well as detection of organo-biomolecules because of their enhanced electrical properties ${ }^{[4]}$. Moreover, the nanocomposites with magnetic nanoparticles provide a broad spectrum in the field of biomedical engineering, cancer therapy and sensing etc ${ }^{[5]}$. In addition, embedding of these functional nanomaterials in some suitable matrix such as polymer is another effective way to enhance their properties as well as protect the nanomaterials from the outer environment to carry on their functionality and stability for a longer period ${ }^{[3]}$. The polymer based coating with multifunctional behavior would be very interesting and demanding in the field of sensors and antibacterial coating that can also be applicable in other field of solar energy and environmental applications. I hope to see new advancement in this field through this journal and request to the authors to conduct more research on multifunctional nanomaterials including other nanomaterials besides mentioned above with increased functional and tunable properties, greater stability and higher reproducibility.

The journal of nanotechnology and materials science is devoted to focus on such an interdisciplinary research including physics, chemistry and biological nanomaterials for specific and multifunctional applications and welcome the articles based on this theme. This will take the research in multidisciplinary aspect including all fields of sciences and provide a comprehensive research database of these nanomaterials.

\section{References}

1. Prakash, J., Kumar, P., Harris, R.A., et al. Synthesis, Characterization and Multifunctional Properties of Plasmonic Ag- $\mathrm{TiO}_{2}$ Nanocomposites. (2016) Nanotechnology 27(35): 355707.

2. Prakash, J., Harris, R.A., Swart, H.C. Embedded plasmonic nanostructures; synthesis, fundamental aspects and their SERS applications. (2016) International Reviews in Physical Chemistry 35(3): 353-398.

3. Prakash, J., Pivin, J.C., Swart, H.C. Noble metal nanoparticles embedding in polymeric materials: Fundamentals to Applications. (2015) Adv Colloid Interface Sci 226: 187-202.

4. Georgakilas, V., Jitendra, N.T., Christian, K.K., et al. Noncovalent Functionalization of Graphene and Graphene Oxide for Energy Materials, Biosensing, Catalytic, and Biomedical Applications. (2016) Chemical Reviews 116(9): 5464-5519.

5. De Crozals, G., Bonnet, R., Farre, C., et al. Nanoparticles with multiple properties for biomedical applications: A strategic guide. (2016) Nano Today 11(4): 435-463.
Ommega Online Publishers

Journal Title: Journal of Nanotechnology and Materials Science Journal Short Name: J Nanotech Mater Sci
Journal ISSN: 2377-1372

E-mail: nanoscience@ommegaonline.com

Website: www.ommegaonline.org 